\title{
Das älteste Pleistozän im Illergletscher-Vorland - Neue Ergebnisse zur Stratigraphie und Paläogeographie der Biberkaltzeit im schwäbischen Alpenvorland
}

\author{
RAIMO BECKER-HAUMANN*)
}

\author{
Biberglaciation, Bavarian Alpine Foreland, Illerglacier, Iller-Lech-Platte, Stratigraphy of \\ the Oldest Pleistocene, Paleogeography, Fluvial History \\ of Iller river and Lech river, Palaeoclimatology
}

\begin{abstract}
Kurzfassung: Das Vorland des Illergletschers (bayerisch-schwäbisches Alpenvorland) ist dadurch gekennzeichnet, daß sich fluviatile Terrassen aus dem ältesten Pleistozän (Biber- und Donaukomplexe) in ausgedehnten Vorkommen erhalten haben. Für diese auf der mittleren und östlichen Iller-Lech-Platte auftretenden Ablagerungen wird ein neues stratigraphisches Konzept vorgestellt, das die Korrelation bisher isoliert betrachteter Terrassenvorkommen ermöglicht. Für die Rekonstruktion der fluß- und landschaftsgeschichtlichen Entwicklung des Illergletscher-Vorlandes ergeben sich dadurch neue Ansatzpunkte.

Aus der Biberkaltzeit sind fünf, aus der nachfolgenden Donaukaltzeit vier Terrassen von Iller und Lech nachzuweisen. Aufgrund der Sedimentausbildung werden kaltzeitliche Bedingungen mit Terrassenakkumulation dokumentiert, die sich mehrfach mit wärmeren Phasen, die zu dominierender Tiefenerosion führten, abwechselten. Während der Biberkaltzeit verlief die Iller von Kempten über Mindelheim und Augsburg in einem bis $30 \mathrm{~km}$ breiten Tal zur Donau und wechselte bis zur jüngeren Donaukaltzeit in ein weiter westlich gelegenes Tal. Der Lech ist aus der Donaukaltzeit mit drei Terrassen entlang seines heutigen Unterlaufes überliefert.
\end{abstract}

\section{[The oldest Pleistocene of the Illerglacier Foreland - Results on stratigraphy and paleogeography of the Biberglaciation of Bavarian Alpine Foreland]}

Abstract: One peculiarity of the Illerglacier Foreland
(Bavarian Alpine Foreland) is the widespread occur-
rence of fluvial terraces of the oldest Pleistocene (Biber-
and Donaucomplex). In this paper a new stratigraphy
for the middle and eastern Iller-Lech-Platte with these
stratigraphic units is developed allowing the correlation
of several gravel terraces regarded before as isolated
occurrences. These results lend support to the recon-
struction of the fluvial history of the survey area.
Five terraces of Biber- and four of the Donaucomplex

*) Anschrift des Verfassers: Dr. R. Becker-Haumann, Geologisches Institut der Universität Köln, Abteilung Quartärgeologie, Zülpicher Str. 49a, D-50674 Köln, email: r.behaumann@uni-koeln.de deposited from rivers Iller and Lech can be distinguished. Coldclimatic conditions are documented by the habitus of the sediments whereas the warmer times lead to vertical erosion, producing the edges of the distinct terraces. During the Bibercomplex the Iller river flows from Kempten, Mindelheim, Augsburg to the Donau river in a valley up to $30 \mathrm{~km}$ wide. Before the younger Donaucomplex the Iller river changed its valley to a more northwestern one. The Lech river is documented by three terraces of Donau-age preserved in the now-a-days lower reach of Lech river.

\section{Einleitung}

Das zwischen den Flüssen Iller und Lech gelegene Illergletscher-Vorland wird in Alpennähe durch mindel- bis würmzeitliche Moränen der alpinen Auslaßgletscher geprägt, an die sich nach Norden bis zur Donau fluviatile und glazifluviatile Schotterterrassen auch älterer Kalt- und Eiszeiten anschließen. Diese lassen sich aufgrund ihrer Basishöhen stratigraphisch gliedern, denn bis auf wenige Ausnahmen in Moränennähe liegen nach dem Prinzip der Terrassenstaffelung die älteren Terrassen orographisch oberhalb der jüngeren. Nach den im Westen bzw. im Osten begrenzenden Flüssen wird die Terrassenlandschaft des Illergletscher-Vorlandes als Iller-Lech-Platte bezeichnet.

Die vorliegende Arbẹit behandelt die ältesten bekannten fluviatilen Ablagerungen, die durch Alpenflüsse nach der Molasseakkumulation zwischen Iller und Lech geschüttet wurden. Die tertiäre Molasse bildet dabei die Unterlage aller Terrassen und besteht aus unverfestigten Klastika, die unter teils marinen, teils terrestrischen Bedingungen aus den Alpen in das Molassebecken geschüttet wurden (LEMCKE 1988, dort weiterführende Literatur, Bachmann \& Müller 1996). Das Untersuchungsgebiet umfaßt die Staudenplatte, Staufenberg-Terrassentreppe, einen Teil der Aind- 
linger Terrassentreppe sowie den Arlesrieder Schotter nebst einiger benachbarter Schotterkuppen (Abb. 1), auf denen sich Reste biberzeitlicher Illerterrassen erhalten haben.

Das bayerisch-schwäbische Alpenvorland ist das klassische Gebiet der alpinen Quartärforschung, in dem bereits PENCK \& BRÜCKNER (1909) mit der terrassenstratigraphisch begründeten Gliederung pleistozäner Ablagerungen in Günz, Mindel, Riß und Würm die Grundlagen der modernen Quartärstratigraphie legten. Nachdem EBERL (1930) mit der Donaukaltzeit die nächst ältere Akkumulationsphase zur Günzkaltzeit auf der Iller-Lech-Platte beschrieben hatte, gelang es ScHAEFER (1957), noch ältere biberzeitliche Terrassenreste auf der Staufenberg-Terrassentreppe zu identifizieren. Graul (1949) gliederte die Iller-Lech-Platte aufgrund geomorphologischer Kriterien in Teilplatten, die er paläogeographisch interpretierte, indem er das heutige Dinkelscherbener Becken als vormaliges Nordwestufer des Wertach-MindelSystems mit der Funktion einer Altwasserscheide ansah. Der Begriff der "Eiszeitengruppe“, der bis heute unter der Bezeichnung „Komplex“ Gültigkeit besitzt, wurde ebenfalls von EBERL (1930) eingeführt. Die Staudenplatte wurde von Schaffer $(1953,1957)$ in zwei süd-nord-verlaufende Schotterstränge gegliedert, die von der Wertach aus südlicher Richtung geschüttet worden sein sollen. Der Eisrand soll aufgrund der Vergröberung des Schotters nach Süden und der sich in gleicher Richtung versteilenden Schotterbasis bis knapp südlich der Staudenplatte gereicht haben, was bereits von SINN (1971) anhand detaillierterer Geländeaufnahmen bestritten wurde. SINN (1972) gliederte den Arlesrieder Schotter in Vorkommen zweier süd-nord-verlaufender Rinnen, denen von RÖGNER (1986) auch der isoliert gelegene Hochfirst als südlichster Schotterrest zugeordnet wurde. Die Iller-Mindel-Platte wurde von Jerz et al. (1974) in einer Geologischen Übersichtkarte (GÜK 100) dargestellt, auf der Arlesrieder Schotter und Staudenplatte als jeweils einheitliche Akkumulationen ausgewiesen sind. Mit dem Aufbau der östlich des Lechtales gelegenen Aindlinger Terrassentreppe haben sich Graul (1943), SCHAEFer (1966) und zuletzt Tillmanns \& Brunnacker \& LÖscher (1983) beschäftigt. Letztere Autoren legten eine GK 50 vor, auf der die Terrassentreppe in einen Hochschotter, drei Deckschotter, eine Hochterrasse sowie mehrere Zwischenterrassen gegliedert ist, wobei eine Verknüpfung der Deck- schotter mit höhengleichen Terrassen auf der östlichen Iller-Lech-Platte von BECKER-HAUMANN (1995 a) vorgeschlagen wurde.

\section{Untersuchungsmethoden}

Die im Gelände anhand von Aufschlüssen oder Bohrungen (Rammkernbohrungen und 1-mPürckhauer-Bohrungen) ermittelte Schottersohle wurde mit einem barometrischen Thommen-Altimeter eingemessen. Die im hier vorgestellten Arbeitsgebiet insgesamt ermittelten mehr als 300 Werte besitzen durch Luftdruckschwankungen während der Messungen und der Gerätetoleranz eine Genauigkeit von $\pm 5 \mathrm{~m}$.

Zur Ermittlung der Kornverteilung wurden mit einem Holzkastensiebsatz der DIN 4187/2 ca. 25 kg bergfeuchtes Sediment gesiebt und die Mengenanteile der Fraktionen $63 \mathrm{~mm}, 35,5 \mathrm{~mm}, 20 \mathrm{~mm}$, $11,2 \mathrm{~mm}$ und $6,3 \mathrm{~mm}$ ermittelt. Die Kornfraktion $6,3 \mathrm{~mm}$ wurde im Labor naß gesiebt und die Ergebnisse beider Siebungen zu einer Körnungslinie verrechnet.

An durchschnittlich 300 Geröllen der Korngröße 20 - $63 \mathrm{~mm}$ wurden die petrographische Zusammensetzung bestimmt und zehn verschiedene Gesteinsarten unterschieden, die in Tab. 1 aufgelistet sind.

Um Informationen zur Paläoströmungsrichtung zu erhalten, wurden an möglichst zahlreichen Lokalitäten Messungen der Gerölleinregelungen vorgenommen. In Aufschlüssen mit Nagelfluh (karbonatisch verkitteter Schotter, WeIDENBACH 1974) wurde an ca. 300 plattigen, länglichen Geröllen die Fallrichtung der B-Achse bestimmt. Berücksichtigung fanden dabei nur Gerölle mit einem Einfallen zwischen $10^{\circ}$ und $80^{\circ}$, die mindestens $1 \mathrm{~m}$ oberhalb der Schotterbasis liegen, um Störungen der Hauptströmungsrichtung durch das Relief der Terrassenbasis zu vermeiden. Eine möglichst gleichmäßige Verteilung der Messungen über die Gesamtfläche des aufgeschlossenen Schotters wurde angestrebt. Die Meßwerte wurden in einem radialen Verteilungsdiagramm dargestellt und zeigen ausgeprägte Maxima in Richtung der Paläoströmung. Die Methode beruht darauf, daß sich plattige, längliche Gerölle in einem fließenden Medium mit ihrer längsten Achse (A-Achse) quer zur Strömung ausrichten und die zweitlängste (B-Achse) der Strömung entgegen geneigt ist. Dies stellt die stabilste Lagerung bei minimiertem Widerstand dar.

An durchschnittlich 200 Geröllen der Fraktion 


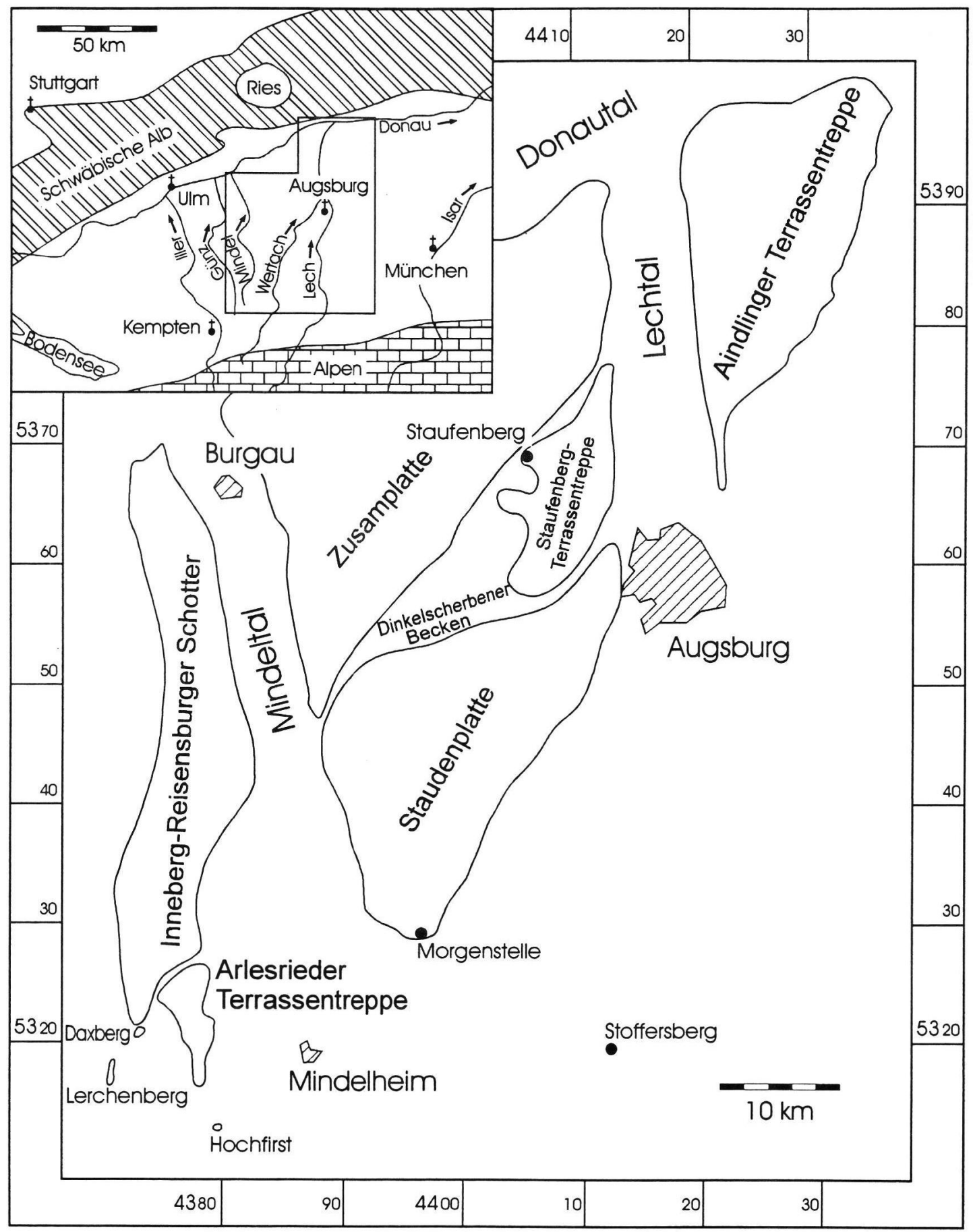

Abb. 1: Übersichtskarte des Untersuchungsgebietes auf der mittleren und östlichen Iller-Lech-Platte mit den verschiedenen Teilgebieten.

Fig. 1: General map of the survey area in the middle and eastern part of the Iller-Lech-Platte with the various sub-areas. 
20-63 mm wurden Rundungsgrade nach REICHELT (1961) mit den vier Gruppen kantig (rauhe Oberfläche, frische, gebrochene Kanten), kantengerundet (teilweise abgerundete Kanten), gerundet (konvexe, unregelmäßige Form) und stark gerundet (regelmäßig runde Form) bestimmt. Aus diesen Angaben wurde der Indexwert Xi nach WINTER (1968) berechnet, der Werte zwischen 10 (kantig) und 40 (stark gerundet) annehmen kann. In jedem geeigneten Aufschluß wurden die zehn größten auffindbaren silikatischen und kalkigen Gerölle nach ihren drei Achsen vermessen. Aus dem Wert der gemittelten B-Achsen ergibt sich für die jeweilige Lokalität eine Information über die Transportkraft des vormaligen Flusses, die mit denen anderer Aufschlüsse verglichen werden kann.

Die schotterbedeckten Anhöhen der StaufenbergTerrassentreppe finden schon bei PENCK \& BRÜCKNER (1909) Erwähnung, der das Gebiet als tektonisch angehobene Scholle deutete. ScHAEFER (1957) legte ein terrassenstratigraphisches Konzept vor und unterschied acht Niveaus, die er altersmäßig den Staudenplatten-Schottern voranstellte.

\section{Terrassenstratigraphische Gliederung}

Die Untersuchungen von Graul (1949) und SchaEFER (1953) zeigten, daß die Schotter der
Staudenplatte ein generelles Basisgefälle nach Norden aufweisen. Es wurde von SCHAEFER als Ergebnis einer Ablagerung aus Süden angesehen bzw. von Graul mit einer Gliederung in verschiedene Terrassen erklärt. Eine Akkumulation aus Süden kann durch die neuen Ergebnisse zur Paläoströmungsrichtung, die nach Nordosten gerichtet gewesen sein muß, ausgeschlossen werden, wie auch kartierbare "Sprünge“ in der Höhenlage der Schottersohlen Südwest-Nordost orientiert sind. Unstetigkeiten der Basishöhen um wenige Meter treten dabei nur lokal auf und gehen auf Rinnen oder Auskolkungen des leicht erodierbaren Molasseuntergrundes zurück. Demgegenüber lassen sich die „Sprünge“ von durchschnittlich $10 \mathrm{~m}$ über die gesamte Fläche der Schotterverbreitung verfolgen und nicht anders als durch Grenzen unterschiedlich hoch liegender Terrassen erklären.

Auf der Staufenberg-Terrassentreppe befinden sich mehrere Schottervorkommen mit einem Höhenunterschied der Basen von insgesamt $28 \mathrm{~m}$ dicht nebeneinander (Pfannenberg bei $542 \mathrm{~m} \mathrm{NN}$, Axtesberg bei $551 \mathrm{~m} \mathrm{NN}$, Staufenberg bei $570 \mathrm{~m}$ NN). Ohne eine Gliederung in unterschiedliche Terrassen würde sich hier eine ursprüngliche Schottermächtigkeit von mehr als $35 \mathrm{~m}$ ergeben, die für ein braided-river System unrealistisch ist und bisher innerhalb eines Aufschlusses auf der

Tab. 1: Der Geröllbestand der biberzeitlichen Illerschotter.

Tab. 1: The petrographic composition of the Biberglacial Illergravels.

\begin{tabular}{|ll}
\hline Kalkstein & meist mikritischer, alpiner Kalk, Fleckenkalk (Lias, Dogger), roter Kalk (Jura) \\
Kalksandstein & $\begin{array}{l}\text { glimmerhaltiger, grünlich-grauer Sandstein, karbonatisch, teils } \\
\text { glaukonititführend (Flysch) }\end{array}$ \\
Kieselskelett & Verwitterungsprodukt von Kieselkalken, scherbig zerfallend \\
Dolomit & alpiner Hauptdolomit \\
Sandstein & grünlich-brauner Glaukonitsandstein (Helvetikum), roter Sandstein (Trias), \\
heller Sandstein & roter und grüner Radiolarit aus dem Jura \\
Hornstein & schwarzer bis dunkelbrauner Hornstein \\
Quarzit & silikatisch verkitteter Sandstein \\
Quarz & stammt aus alpinen Quarzgängen \\
Kristallin & Muskowitgneis, Epidotquarzit, Amphibolit, roter und heller Granit
\end{tabular}


Iller-Lech-Platte nirgends beobachtet wurde. Ebenso wurde für die Aindlinger Terrassentreppe eine Terrassengliederung aufgestellt (Tillmanns \& BRUNNACKER \& LÖSCHER 1983), so daß als Erklärung der "Sprünge“ in den Terrassensohlen der Staudenplatten-Schotter ebenfalls eine Gliederung in mehrere eigenständige Terrassen naheliegend ist. Auf der Staudenplatte lassen sich sechs verschiedene Terrassen unterscheiden, deren fünf höchstgelegenen und damit ältesten den flächenmäßig überwiegenden Teil der Staudenplatte darstellen (Abb. 2). Entlang Südwest-Nordost gerichteter Grenzen ordnen die Terrassen sich von Süden nach Norden mit Höhenunterschieden von durchschnittlich $10 \mathrm{~m}$ untereinander an. Die Terrassengrenzen verlaufen leicht geschwungen und zeichnen die vormaligen südlichen Uferlinien nach. Mit Ausnahme von erhaltenen Prallhangsituationen steigt die Schotterbasis in Richtung auf das vormalige Ufer um einige Meter an. Bei erosiv stark reduzierten und in Einzelvorkommen aufgelösten Terrassen ist das Auskartieren der Schottergrenzen erschwert, doch sind sie im Kartenbild durch Zonen mit Höhenunterschieden bis $10 \mathrm{~m}$ auf Distanzen von wenigen hundert Metern quer zur Paläoströmung kenntlich. Diese Zonen lassen sich in Südwest-Nordost-Richtung über die gesamte Staudenplatte verfolgen und als Terrassengrenzen interpretieren. Im Querprofil (Abb. 3) sind die einstigen Uferpartien der Iller durch lokal auftretendes Einfallen der Schottersohle von stellenweise mehr als $40 \%$ in nördliche Richtung erkennbar, an die sich nach Norden und Süden die nahezu ebenen Terrassenflächen anschließen.

Die Terrassenflächen selbst sind über Entfernungen von mehreren Kilometern entsprechend ihres primären Einfallens von 1,3 bis $2,5 \%$ nach Nordosten geneigt und nur schwach durch Auskolkungen der weichen Molasse durch die geröllbeladenen Wassermassen reliefiert. Solche lokalen Auskolkungen sind für braided river-Systeme charakteristisch, treten stets im Zusammenhang mit flankierenden Geröllbänken auf und sind raschen Veränderungen durch An- und Umlagerung von Sediment unterworfen (FERGUSON 1993). Ein Beispiel findet sich auf der Terrasse B III bei Oberrothan, wo eine $7 \mathrm{~m}$ breite und $3 \mathrm{~m}$ tiefe Auskolkung, die innerhalb des Aufschlusses über eine Distanz von wenigen zehner Metern keine Fortsetzung findet und daher nicht als Rinne interpretiert werden kann, quer angeschnitten ist (Abb. 4).

Die älteste Terrasse B I befindet sich auf der Morgenstelle an der Südspitze der Staudenplatte mit einer Basishöhe von etwa $651 \mathrm{~m} \mathrm{NN}$ (Abb. 2). Erosiv wurde das Vorkommen auf eine Mächtigkeit von 3,1 m reduziert (AKTAS \& BeCKer-HaUMANN 1991) und chemisch stark zersetzt. Die Grobheit des Schotters, seine Lage auf der topographisch höchsten Stelle der Staudenplatte sowie zahlreiche Kieselskelette als Verwitterungsprodukte kieseliger Kalksteine weisen das Material im Widerspruch zu SinN (1971), der das Material als Fließerde beschrieb, als insitu liegenden Terrassenschotter aus. Die Beobachtung, daß der Morgenstelle-Schotter auf der gesamten Fläche seiner heutigen Verbreitung von etwa 150 x 150 $\mathrm{m}$ horizontal liegt, schließt seine Deutung als Randlage der sich nördlich anschließenden Terrasse B II aus. Der innerhalb des Lechtales gelegene Stoffersberg ist mit einem etwa $10 \mathrm{~m}$ mächtigen Schotter bedeckt, der mit seiner Basis auf $680 \mathrm{~m}$ NN liegt. Wegen seiner isolierten Position muß eine Verknüpfung mit einer der ältesten Terrassen auf der Staudenplatte wie sie von RÖGNER (1986) vorgeschlagen wurde jedoch unsicher bleiben.

Die Terrassen B II und B V sind trotz ihrer Zerlegung durch die Bachtäler in Süd-Nord-orientierte Hügelzüge besser erhalten als der MorgenstelleSchotter. Die heutige Breite der Vorkommen beträgt 3 bis $5 \mathrm{~km}$, und vielfach ist der Schotter in einer Mächtigkeit von 10 bis $12 \mathrm{~m}$ lößlehmbedeckt erhalten, wobei auch wenig verwittertes Sediment mit Anteilen der Karbonatgerölle bis $90 \%$ vorkommt. Dolomit als sehr schnell verwitterndes Gestein ist nur in wenigen Prozenten als Geröll erhalten, im Nagelfluh lassen sie sich in zerfallenem, sog. veraschtem Zustand (FEzER 1969) bis etwa $30 \%$ nachweisen. Als silikatische Gerölle finden sich Sandsteine verschiedener Art mit 4\%, Radiolarite, Hornsteine, Quarzite, Gangquarze und Kristalline mit zusammen $6 \%$ (Tab. 2). Unter letzteren können Gneise unterschiedlicher Ausbildung, Epidotquarzite, rote und helle Granite, Diorite und Amphibolite unterschieden werden (Becker-Haumann 1996), die durch Transfluenzen aus den Zentralalpen geliefert wurden. Die petrographische Zusammensetzung der Terrassen B I bis B V ist einheitlich und belegt ein Herkunftsgebiet in den nördlichen Kalkalpen.

Auf der westlichen Staudenplatte ist zwischen den Terrassen B III und B IV ein weiteres Terrassenniveau eingeschaltet (Abb. 2, 3), das einzig im Umkreis der Ortschaften Haselbach und Gessertshausen zu finden ist und daher als B III a bezeichnet wird. Bei der zumeist trimodalen Kornverteilung der Schotter dominiert Mittel- bis Grob- 


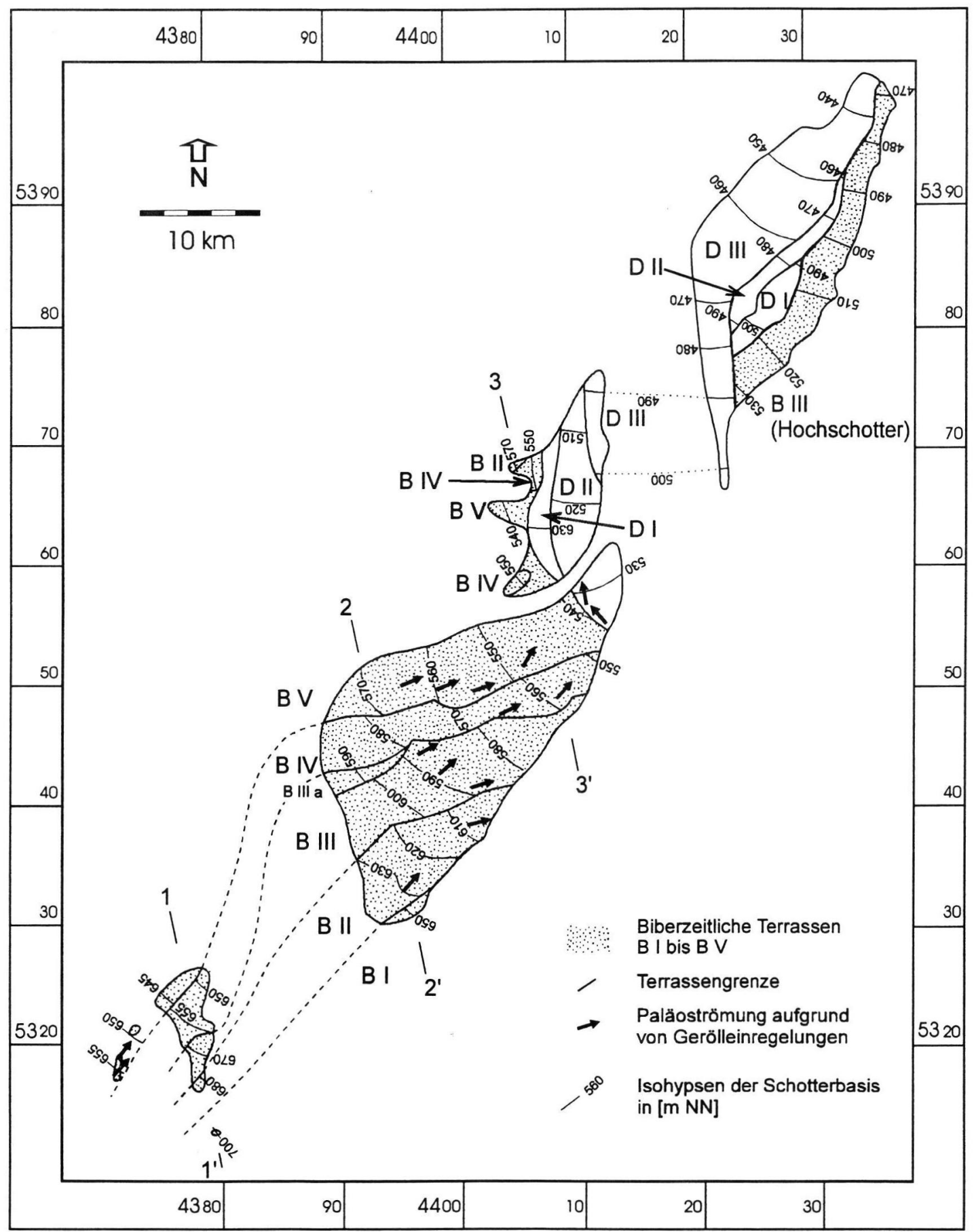

Abb.2: Stratigraphische Gliederung der biberzeitlichen Illerterrassen auf der mittleren und östlichen Iller-Lech-Platte. Die Profile sind in Abb. 3 dargestellt.

'Fig. 2: Stratigraphic subdivision of the Biberglacial Illerterraces of middle and eastern Iller-Lech-Platte. The cross-sections are shown in Fig. 3. 


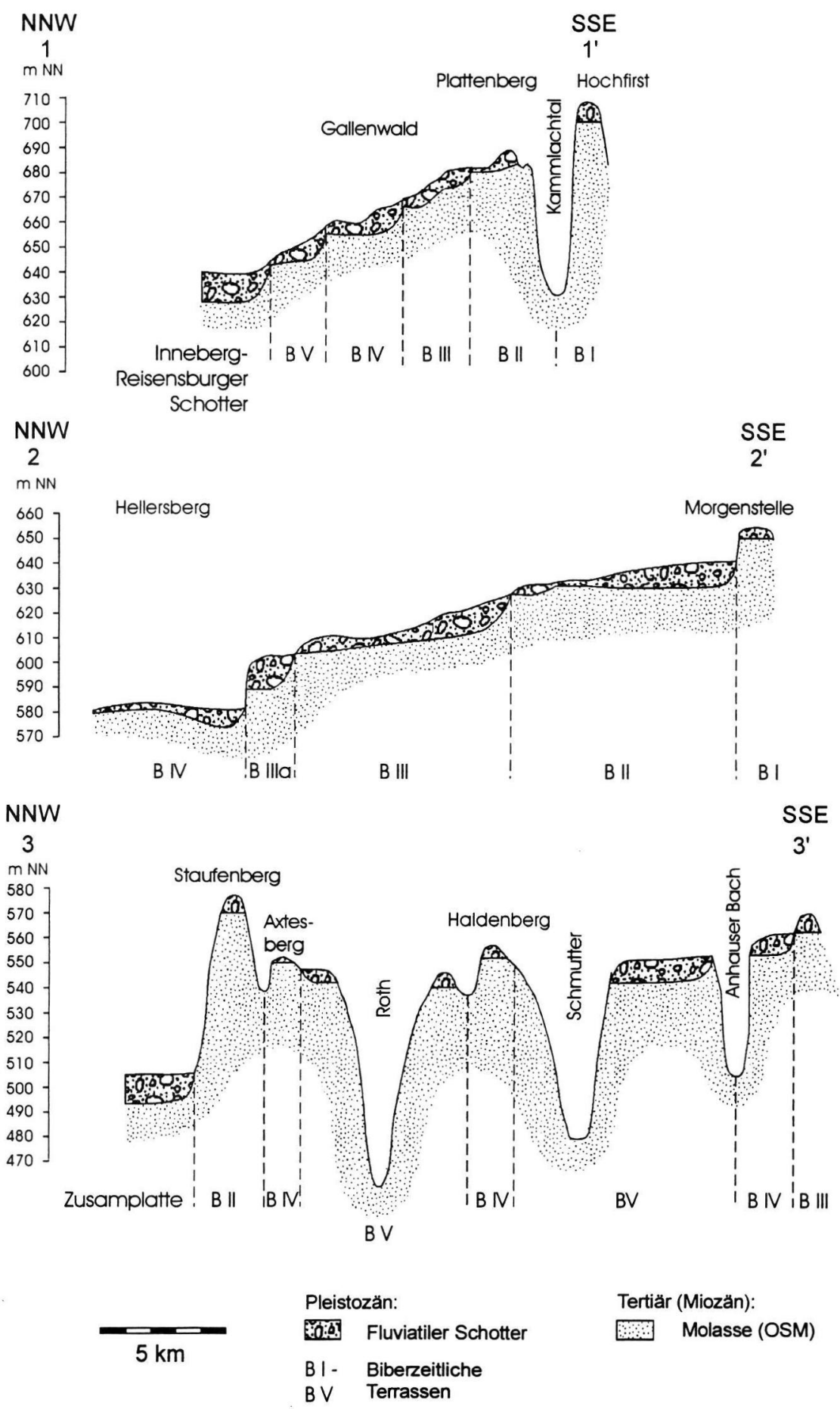

Abb. 3: Schematische Querprofile durch Arlesrieder Schotter (1-1'), die Staudenplatte (2-2') und die Staufenberg-Terrassentreppe mit Staudenplatte (3-3'). Periglaziale Deckschichten sind nicht eingetragen. Lage der Profile in Abb. 2.

Fig. 3: Schematic cross-sections of Arlesrieder Schotter (1-1'), Staudenplatte (2-2') and Staufenberg-Terrassentreppe and Staudenplatte (3-3'). Periglacial coversediments are not shown. Position of cross-sections are shown in Fig. 3. 
NE

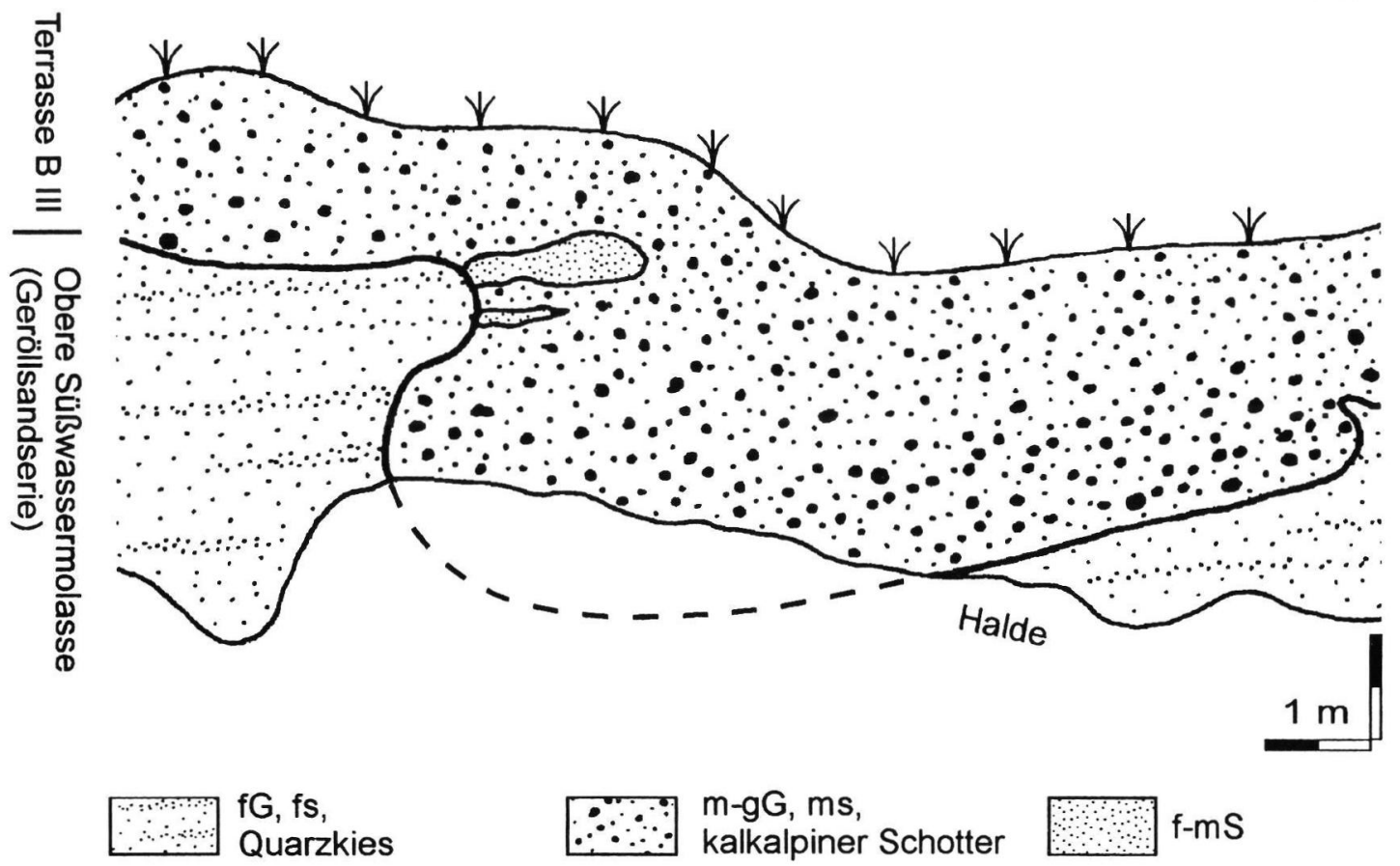

Abb. 4: Profil der (Kgr.) Oberrothan mit dem Querschnitt einer Auskolkung an der Basis der Terrasse B III. Fig. 4: Cross-section of a pool in the basal zone of Terrace B III in the gravel pit Oberrothan.

kies $(6,3-63 \mathrm{~mm})$ mit 50 - $70 \%$, ein zweites Maximum findet sich im Fein- bis Mittelsand durch Aufarbeitung des Molassesandes, ein weiteres im Feinkies durch Umlagerung kiesiger Molasse der Geröllsandserie sensu Doppler (1989). Die Schotter sind stets schlecht sortiert, im Dezimeter- bis Meterbereich horizontal gebankt, Rinnenstrukturen und Sandlinsen finden sich nur vereinzelt. Als Hinweis auf stark schwankende Wasserführung und stetige Zufuhr von Sand durch Aufarbeitung der Molasse dominiert matrixgestützter Schotter ohne Schrägschichtung, in den nur untergeordnet besser sortierte Lagen aus Gerüstschotter eingeschaltet sind.

Über die gesamte, bis heute dokumentierte Laufstrecke der biberzeitlichen Iller von etwa $100 \mathrm{~km}$ ist keinerlei Verfeinerung des Sediments festzustellen. Bei der Kornverteilung bleibt der Anteil des Mittel- bis Grobkieses, der aus den Alpen geliefert wurde, mit 60 bis $70 \%$ gleich, wie sich auch die Ausmaße der größten Gerölle im Talverlauf nicht ändern. Sie liegen im Mittel der B-Achse der zehn größten Karbonatgerölle bei $9-12 \mathrm{~cm}$, bei Silikatgeröllen bei 10 - $14 \mathrm{~cm}$. Diese Befunde deuten auf sehr wechselhafte Abflußverhältnisse mit (saisonal ?) starken Fluten, die auch Grobmaterial bis zum Vorfluter transportieren konnten. Der Rundungsgrad Xi beträgt im Mittel der bearbeiteten Aufschlüsse zwischen 30 und 35, ist jedoch petrographieabhängig, indem die Werte mit zunehmender Verwitterungsresistenz abnehmen. Wegen der hohen Gehalte kalkiger Gerölle spiegelt der Wert im wesentlichen die Rundung dieser Komponenten wider.

Die Ergebnisse der Einregelungsmessungen belegen eine Strömung aus südwestlicher Richtung (Abb. 2), die durch die Nähe zum einstigen Ufer oder lokale Faktoren der Rinnenmorphologie um $\pm 30^{\circ}$ beeinflußt sein kann. Die Maxima der meisten Verteilungsdiagramme sind jedoch deutlich und dokumentieren eine den Terrassengrenzen parallel verlaufende Paläoströmungsrichtung aus etwa $250^{\circ}$. Neben der Petrographie der Gerölle und der Ausrichtung der Terrassengrenzen ist dieses Ergebnis ein wichtiger Beleg für die Iller als ablagernder Fluß der Terrassen B I bis B V, denn die Richtung weist von der Staudenplatte nach Memmingen ins heutige obere Illertal. Da die großen Täler der nördlichen Alpen präpleistozän angelegt sind (HANTKE 1993), wird auch die biberzeitliche Iller von Kempten ihren Ausgang genommen haben. Auf dem westlich von Mindel- 
Tab. 2: Die petrographische Zusammensetzung der biberzeitlichen Illerschotter anhand ausgewählter Kiesgruben mit möglichst unverwittertem Material. Von der Staufenberg-Terrassentreppe konnten nur stark verwitterte Proben k'wonnen werden.

Tab. 2: Petrographical composition ob the Biberglacial Illerterraces from selected locations with far fresh sediment as possible. From the Staufenberg-Terrassentreppe only roughly weathered samples could be recovered.

Gesteine:

Lokalitäten:

$A=$ Arlesrieder Terrassentreppe

$S=$ Staudenplatte

ST = Staufenberg-Terrassentreppe

\begin{tabular}{|l|l}
\hline Terrasse B I & \\
\hline (Kgr.) Hochfirst (A) & 8 \\
\hline Terrasse B II & \\
\hline (Kgr.) Markt Wald (S) & 8 \\
\hline (Kgr.) Angelberger Forst (S) & 9 \\
\hline (Kgr.) Schellenberg (S) & 8 \\
\hline (Kgr.) Plattenberg (A) & 8 \\
\hline Staufenberg (Aufsammlung) (ST) & 0 \\
\hline Terrasse B III & \\
\hline (Kgr.) Lenzenberg (S) & 9 \\
\hline (Kgr.) Ziegelberg (S) & 8 \\
\hline Schurf Schorenwald (A) & 8 \\
\hline Terrasse B IV & \\
\hline (Kgr.) W Kreuzanger (S) & 8 \\
\hline (Kgr.) NE Reinhartshausen (S) & 8 \\
\hline (Kgr.) NE St. Johann (A) & 8 \\
\hline (Kgr.) S Rommelsried (ST) & 0 \\
\hline Terrasse B V & \\
\hline (Kgr.) SE Fischach (S) & 85 \\
\hline (Kgr.) SE Bauhofen (S) & 88 \\
\hline (Kgr.) W Wollmetshofen (S) & 90 \\
\hline (Kgr.) Sparloh (S) & 85 \\
\hline (Kgr.) Holzarawald (S) & 82 \\
\hline (Kgr.) S Arlesried (A) & 83 \\
\hline (Kgr.) Steineberg W Biburg (ST) & 0,0 \\
\hline
\end{tabular}

\begin{tabular}{|c|c|c|c|c|c|c|c|c|c|c|}
\hline 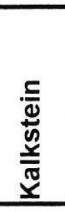 & 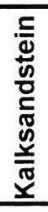 & 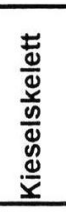 & $\begin{array}{l}\text { 믈 } \\
\text { 응 } \\
\end{array}$ & $\begin{array}{l}\frac{5}{\bar{d}} \\
\frac{0}{0} \\
\frac{\pi}{\pi} \\
0\end{array}$ & $\begin{array}{l}\text { 紊 } \\
\frac{\pi}{0} \\
\frac{0}{0} \\
\mathbb{0} \\
\mathbb{x}\end{array}$ & $\begin{array}{l}\text { 듬 } \\
\text { 苋 } \\
\text { 돈 } \\
\text { 우 }\end{array}$ & $\begin{array}{l}\frac{\pi}{N} \\
\text { 预 } \\
0\end{array}$ & $\begin{array}{l}\text { N } \\
\text { 胥 } \\
\text { O }\end{array}$ & 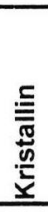 & 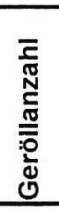 \\
\hline 80,0 & 1,2 & 4,1 & 3,5 & 3,0 & 0,6 & 0,0 & 2,3 & 0,6 & 4,7 & 173 \\
\hline & & & & & & & & & & \\
\hline 84,2 & 1,6 & 0,9 & 1,6 & 3,0 & 0,2 & 1,4 & 1,4 & 2,1 & 3,6 & 430 \\
\hline 90,7 & 0,5 & 0,0 & 1,3 & 2,7 & 0,0 & 1,3 & 0,8 & 1,9 & 0,8 & 371 \\
\hline 88,6 & 1,7 & 0,0 & $\overline{1,1}$ & 2,7 & 0,6 & $\overline{1,4}$ & 1,4 & 1,9 & 0,6 & 360 \\
\hline 88,9 & 0,6 & 0,9 & 1,5 & 3,0 & 0,6 & 0,3 & 2,1 & 1,5 & 0,6 & 331 \\
\hline 0,0 & 4,1 & 39,7 & 0,0 & 10,5 & 1,8 & 2,3 & 28,1 & 13,5 & 0,0 & 171 \\
\hline 915 & & & 10 & 23 & 08 & 08 & 04 & ח & 23 & 265 \\
\hline 85,6 & 0,6 & 0,4 & 0,0 & 1,2 & 0,2 & 3,2 & 2,7 & 2,3 & 3,8 & 476 \\
\hline 89,2 & 1,1 & 1,4 & 6,1 & 1,1 & 0,0 & 0,0 & 0,4 & 0,7 & 0,0 & 278 \\
\hline & & & & & & & & & & \\
\hline 84,6 & 4,4 & 0,0 & 1,8 & 2,6 & 1,3 & 0,9 & 0,9 & 1,4 & 2,1 & 704 \\
\hline 88,3 & 1,6 & 0,0 & 0,9 & 0,5 & 0,9 & 0,9 & 2,8 & 0,9 & 3,2 & 435 \\
\hline 87,5 & 0,0 & 14 & 6,2 & 1,4 & 0,3 & 0,3 & 0,3 & 0,6 & 2,0 & 356 \\
\hline 0,0 & 0,0 & 47,6 & 0,0 & 6,2 & 2,0 & 7,5 & 27,2 & 9,5 & 0,0 & 147 \\
\hline & & & & & & & & & & \\
\hline 85,5 & 2,2 & 0,2 & 2,4 & 2,9 & 0,4 & 1,3 & 1,3 & 1,6 & 2,2 & 451 \\
\hline 88,0 & 0,7 & 0,0 & 0,0 & 2,3 & 0,5 & 1,8 & 30 & 2,3 & 1,4 & 440 \\
\hline 90,1 & 0,6 & 0,2 & 0,0 & 1,0 & 0,4 & 0,8 & 4,7 & 0,4 & 1,8 & 506 \\
\hline 85,2 & 1,0 & 0,0 & 0,0 & 3,1 & 0,5 & 2,4 & 1,4 & 2,4 & 4,0 & 417 \\
\hline 82,7 & 2,3 & 0,0 & 1,2 & 3,3 & 0,9 & 1,9 & 35 & 1,6 & 2,6 & 430 \\
\hline 83,6 & 1,6 & 0,0 & 9,6 & 1,0 & 0,5 & 0,5 & 1,1 & 0,0 & 2,1 & 188 \\
\hline 0,0 & 0,0 & 51,3 & 0,0 & 6,0 & 0,0 & 3,5 & 28,7 & 9,6 & 0,9 & 115 \\
\hline
\end{tabular}

heim gelegenen Arlesrieder Schotter sind ebenfalls die Terrassen B I bis B V wiederzufinden, die sich von Süden nach Norden mit Höhenabständen von jeweils 8 bis $10 \mathrm{~m}$ untereinanderstaffeln. Die höchstgelegene Terrasse B I hat sich auf dem Hochfirst - einer isolierten Anhöhe südlich des Arlesrieder Schotters - in einer Mächtigkeit von etwa $7 \mathrm{~m}$ mit einer Basishöhe von 697 - 700 m NN erhalten. $2,5 \mathrm{~km}$ weiter nördlich setzt am Plattenberg die nächst tiefer gelegene Terrasse B II mit einer Sohlhöhe von $681 \mathrm{~m}$ NN ein. Der im Vergleich zu den Abständen zwischen den Terrassen B II bis B V deutlich größere Niveauunterschied von 16 bis $19 \mathrm{~m}$ von B I zu B II stellt eine Parallele zur Staudenplatte dar. Dort beträgt der Abstand vom Morgenstelle-Schotter B I zur B II-Terrasse 16 bis $18 \mathrm{~m}$. Aufgrund der Gliederung in eine Anzahl von Terrassen kann bei Arlesrieder Schotter und Hochfirst in Analogie zu Staufenberg- und Aindlinger Terrassentreppe ebenfalls von einer Terrassentreppe gesprochen werden.
Die quartären Ablagerungen der Arlesrieder Terrassentreppe weisen eine vergleichbare petrographische Zusammensetzung und gleiche sedimentologische Eigenschaften wie die StaudenplattenSchotter auf (Becker-Haumann 1995 b). Die Terrassen $\mathrm{B} I$ bis $\mathrm{B} \mathrm{V}$ lassen sich mit einem Gefälle von 2,6 bis 3,4\%o mit den äquivalenten Niveaus der Staudenplatte verbinden, wobei sich im Vergleich zu den dort berechneten 1,3 bis 2,5\%o eine Versteilung des Tales stromaufwärts dokumentiert. Auch die rekonstruierte Paläoströmungsrichtung und die Ausrichtung der Terrassengrenzen legen eine Verknüpfung von Arlesrieder Terrassentreppe und Staudenplatte nahe (Abb. 2)

Der im Günztal gelegene Lerchenberg und ein Vorkommen am Ort Daxberg wurden bislang den donauzeitlichen Unteren Deckschottern zugerechnet, doch legen neue Kartierergebnisse nahe, beide Vorkommen mit der Terrasse B V zu verbinden, in deren direkter südwestlicher Verlängerung sie sich befinden (Becker-Haumann 1997). 
Die am Lerchenberg vorgenommenen Einregelungsmessungen sowie die rekonstruierbaren Gefällswerte machen eine Verknüpfung mit der Terrasse B V wahrscheinlicher als mit den Unteren Deckschottern des Inneberg-Reisensburger Schotters (LÖsCHER 1976).

Die Staufenberg-Terrassentreppe wird von einer Anzahl unterschiedlich hoch liegender Terrassen aufgebaut, die in erosiv stark reduzierten Vorkommen erhalten sind. Von SCHAEFER (1957) wurden sie in die Terrassen äd 1 bis äd 8 untergliedert und altersmäßig den Staudenplatten-Schottern vorangestellt, wobei die Gliederung von ScHEuENPFLUG (1994) in Einzelheiten modifiziert wurde. Eine Neubearbeitung der Region zeigte, daß sich die im Westen befindlichen Terrassenreste mit verschiedenen Terrassen auf der Staudenplatte korrelieren lassen (BECKFr-HALMANN 1995 b), da sie mit ihren Basen höhengleich liegen (Abb. 2). Der am höchsten gelegene Staufenberg bei der Ortschaft Bonstetten ist bei Berücksichtigung des primären Einfallens nach Nordosten mit der Terrasse B II der südlichen Staudenplatte zu verknüpfen, die sich im Süden des Staufenberges anschließenden Schottervorkommen entsprechen den Terrassen B IV und B V.

Während auf Staudenplatte und Arlesrieder Schotter nur die Südflanken des früheren Illertales erhalten sind, sind am Staufenberg und am unweit südlich gelegenen Axtesberg die einzigen Stellen mit Resten des Nordufers vorhanden. Für die B II-Zeit läßt sich daraus an dieser Stelle eine Talbreite von 25 bis $30 \mathrm{~km}$, für die B IV-Zeit eine solche von $20 \mathrm{~km}$ und für die B V-Zeit eine Breite von $15 \mathrm{~km}$ ableiten. Die Iller hat sich in diesem Talabschnitt folglich während der Biberkaltzeit schrittweise in ihre eigenen Terrassen eingetieft. Das Vorkommen der Terrasse B IV befindet sich am Haldenberg innerhalb der Fläche von Terrasse B V und läßt auf eine Insel inmitten des biberzeitlichen Illertales schließen. Trotz der Verengung des Tales im Vergleich zu den durch die älteren Terrassen dokumentierten Talverläufen manifestiert sich durch die Erhaltung der Insel eine Verringerung der erosiven Kraft der Iller. Gleiches belegen die Daten der größten Gerölle, deren über die gesamten Terrassen berechneten Mittelwerte von Terrasse B II zu B V um $3 \mathrm{~cm}$ abnehmen (von Terrasse B I liegt zu wenig Material vor),

Auf der östlich des Lech gelegenen Aindlinger Terrassentreppe stellt der Hochschotter die älteste erhaltene stratigraphische Einheit dar (Abb. 2), die mit 1,6\% nach Nordosten einfällt (Tillmanns
\& BRunNaCker \& LÖscher 1983). Bei einer Rekonstruktion des Talverlaufes der biberzeitlichen Iller zeigt sich, daß der Hochschotter inmitten des nach Nordosten verlängerten Tales liegt. Bereits HABBE \& RÖGNER (1989) vermuteten eine Ablagerung aus südöstlicher Richtung. Bei Berücksichtigung des Einfallens läßt sich der Hochschotter mit gewisser Unsicherheit aufgrund der weiten Entfernung, über die interpoliert werden muß, mit der Terrasse B III mit einem Gefälle von 1,7\% verbinden und als nordöstlichstes Terrassenvorkommen der biberzeitlichen Iller identifizieren.

Die östlichen Bereiche der Staufenberg-Terrassentreppe werden von drei Terrassen D I bis D III eingenommen, die die nächst jüngeren fluviatilen Akkumulationen zur Terrasse B V darstellen, da sie in diese eingeschnitten sind und sich in direktem Kontakt ohne Zwischenniveaus zu ihr befinden. Die Terrasse D II ist als einzige auch auf der Nordostspitze der Staudenplatte vorhanden und erlaubt daher die Verknüpfung beider Schotterplatten über das trennende Schmuttertal hinweg. Die Grenzen der Terrassen D I bis D III verlaufen konvex nach Westen ausschwingend dem heutigen Lechtal parallel, auf der nördlichen Staudenplatte biegt der Rand der Terrasse D II nach Südosten ab. Hier konnten an zwei Lokalitäten Messungen der Gerölleinregelungen vorgenommen werden, deren Ergebnisse eine Vorzugsrichtung der Paläoströmung parallel zum kartierten Terrassenrand nach Nordwesten ergaben (aufgel. Kgr. Bergheim: $130^{\circ}$, aufgel. Kgr. Prügelkopf: $150^{\circ}$ ).

Obwohl sich keine geröllpetrographischen Unterschiede zu den biberzeitlichen Illerschottern nachweisen lassen, dürften die Terrassen D I bis D III entgegen der Annahme von z. B. ScheuenPFLUG (1986) von einem Ur-Lech und nicht von der Iller stammen. Ein Wechsel der Iller aus einem biberzeitlichen Tal, das von Kempten aus nach Nordosten verlief (Terrassen B I bis B V), in ein Ur-Lechtal erscheint unwahrscheinlich, da die Iller zur Ablagerung der drei fraglichen Terrassen alpenparallel über orographisch recht hoch liegendes Gelände hätte verlaufen müssen. Zur Akkumulation der nächst jüngeren Unteren Deckschotter (D IV) hätte sie anschließend von Kempten aus erneut nach Norden schwenken müssen. Leitgerölle für den riß- und würmzeitlichen Lech wie der grüne Julier-Granit und der graue, fein geäderte Hauptdolomit fehlen in den Terrassen D I bis D III, jedoch müssen die Liefergebiete für den Lech im Unterpleistozän nicht die gleichen wie im Mittel- und Oberpleistozän gewesen sein. Aufgrund gleicher Höhenlage lassen sich die be- 
schriebenen Terrassen D I bis D III mit dem Oberen, Mittleren und Unteren Deckschotter auf der Aindlinger Terrassentreppe sensu Tillmanns \& BRUNNACKER \& LÖSCHER (1983) verknüpfen (Becker-Haumann 1995 a) und mit einem Gefälle von 1 bis 1,4\% nach Norden fortsetzen. Damit ist der Unterlauf des heutigen Lechtales für die Zeit nach Ablagerung der biberzeitlichen Terrassen B I bis B V und vor der Akkumulation der nächst jüngeren Zusamplatte (Terrasse D IV) mit Terrassen überliefert.

\section{Zur stratigraphischen Einordnung der Terrassen}

Die vorstehend beschriebenen biberzeitlichen Ablagerungen der mittleren und östlichen IllerLech-Platte sind bei Berücksichtigung unterschiedlicher Verwitterungsstadien petrographisch und sedimentologisch einheitlich aufgebaut und zeigen in ihrer Ausbildung große Ähnlichkeit mit jüngeren glazifluviatilen Sedimenten. Vergleichbare Ablagerungsbedingungen können daher vorausgesetzt werden, wobei fehlende zugehörige glaziale Sedimente auf kaltklimatische Bedingungen ohne weitflächige Vereisung des Alpenvorlandes schließen lassen.

Es bestehen hingegen deutliche Unterschiede zu tertiären Schottern der subalpinen Molasse, die geringere Karbonatgehalte bis etwa $50 \%$ aufweisen (EBERHARD 1987) und als Schuttfächer nördlich der Alpentäler zur Ablagerung kamen (ScineMENZ 1960).

Die Terrassen B I bis B V sind aus morphostratigraphischen Gründen als die ersten fluviatilen Terrassen zu bezeichnen, die nach dem Ende der Molassesedimentation im Illergletscher-Vorland akkumuliert wurden, da sich bislang keine noch höher liegenden Terrassen finden ließen. Aufgrund der klimagenetischen Aussage des Materials liegt es nahe, sie als im Pleistozän entstanden anzusehen, wenngleich bereits im Pliozän Anzeichen von Abkühlung auftreten (EBERHARD 1987, HaNTKE 1989). Die einzigen Faunen innerhalb der Staudenplatten-Schotter wurden von Schröder \& Deнm (1951) von der Kiesgrube östlich Fischach beschrieben. Sie bezeugen ein feucht-kühles Klima, wobei sich anhand des Artenspektrums das Alter nur auf Altpleistozän eingrenzen ließ.

Auf der Iller-Lech-Platte konnten bisher nur wenige chronostratigraphische Befunde gewonnen werden, da die Überlieferung im fluviatilen Milieu höchst lückenhaft ist und das hohe Alter zu starker Verwitterung des Sediments führte. Eine in diesem Zusammenhang wichtige Lokalität ist der Uhlenberg auf der Zusamplatte (FILzER \& Scheuenpflug 1970), wo in Decksedimenten nach Kleinsäugerbefunden das jüngere Tegelen (ELLWANGER \& FeJFar \& KoENigSWALd 1994), nach Mollusken wahrscheinlich ebenfalls Tegelen (RÄHLE 1995) dokumentiert ist. Die paläomagnetischen Befunde sind schwer interpretierbar, die normale Magnetisierung im unteren Profilbereich widerspricht jedoch einer Zuordnung zum OlduvaiEvent nicht (STRATtner \& Rolf 1995). Als Mindestalter für die diese Deckschichten unterlagernde Zusamplatte ergibt sich demnach Jung-Tegelen, dem auch die paläomagnetischen Daten vom Deckschichtenprofil Roßhaupten - ebenfalls auf der Zusamplatte gelegen - mit einer überlieferten Brunhes-Matuyama-Grenze (Tillmanns \& Kocr \& BRUNNACKER 1986) nicht widersprechen.

Die Grenze Pliozän / Pleistozän wird in Mitteleuropa bislang noch nicht einheitlich gezogen. International festgelegt wurde diese Grenze am Typprofil Vrica in Kalabrien / Süditalien, in dem oberhalb des Olduvai-Events bei ca. 1,8 Mio. Jahren erstmalig kühlklimatische Faunenelemente auftreten (KRUTzSCH 1988). Im ozeanischen sowie terrestrischen Bereich sind jedoch bereits vorher Indizien für eine Abkühlung nachweisbar, die im obersten Reuver der niederländischen Stratigraphie (BoENIGK et al. 1974, ZAGWIJN 1985) einsetzen. Das Alter dieser Grenze ist etwas jünger als die Gauss-Matuyama-Grenze mit ca. 2,6 Mio. Jahren. Bei Zugrundelegen dieser zweiten Definition ist die Biberkaltzeit in das Prätegelen und/oder das Tegelen zu stellen und repräsentiert damit die älteste Einheit des Pleistozäns im IllergletscherVorland (eine vergleichende stratigraphische $\mathrm{Zu}-$ sammenstellung findet sich in Doppler 1995).

Es wurde vorstehend gezeigt, daß sich auf der östlichen Iller-Lech-Platte eine offenbar lückenlose Folge fluviatiler Akkumulationen erhalten hat, die von den Schottern der Staudenplatte zur vermutlich tegelenzeitlichen Zusamplatte überleiten (Tab. 3). Der Anschluß der Terrasse B V zur Sequenz der Terrassen D I bis D III ist auf der Staufenberg-Terrassentreppe und der nördlichen Staudenplatte gegeben. Die ablagernden Flüsse im ersten Fall die Iller, im zweiten der Lech - sind zwar verschieden, doch sind beide auf die Donau als gemeinsamen Vorfluter eingestellt, womit bei Berücksichtigung der Laufstrecke ein stratigraphischer Vergleich von Terrassen der zwei im Unterpleistozän benachbarten Nebenflüsse möglich ist. Bei dem Ort Biberbach an der Nordspitze der Staufenberg-Terrassentreppe liegt der Zusamplatten-Schotter nur $1 \mathrm{~km}$ entfernt, doch mit seiner 
Tab. 3: Die Terrassengliederung auf der mittleren und östlichen Iller-Lech-Platte. Für die Aindlinger Terrassentreppe sind in Klammern die Benennungen nach TILlMANns \& BRUnNACKer \& LÖsCher (1983), für die Staufenberg-Terrassentreppe nach Becker-Haumann (1995 b) angegeben. Die im Norden von Arlesrieder Terrassentreppe und Staudenplatte befindlichen Vorkommen von Terrasse D IV sind der Vollständigkeit wegen mit angegeben.

Tab. 3: The fluvial terraces of the middle and eastern Iller-Lech-Platte. In brackets the terms of Tillmanns \& BRunNaCKer \& LÖsCher (1983) for Aindlinger-Terrassentreppe, of BeCKer-Haumann (1995 b) for StaufenbergTerrassentreppe are given. The occurrences of terrace D IV in the north of Arlesrieder Terrassentreppe and Staudenplatte are mentioned for completion.

\begin{tabular}{|c|c|c|c|c|}
\hline Stratigraph. Stellung & Arlesrieder-Terrassentreppe & Staudenplatte & Staufenberg-Terrassentreppe & Aindlinger Terrassentreppe \\
\hline \multirow{4}{*}{$\begin{array}{l}\text { Donaukaltzeiten- } \\
\text { Komplex }\end{array}$} & $\begin{array}{l}\text { Terrasse DIV } \\
\text { (Inneberg-Reisensburger Sch.) }\end{array}$ & $\begin{array}{l}\text { Terrasse DIV } \\
\text { (Zusamplatte) }\end{array}$ & & \\
\hline & & & Terrasse D III (Biberbacher T.) & Terrasse D III (u. Deckschotter) \\
\hline & & Terrasse DII & Terrasse D II (Bergh.-Ayst. T.) & Terrasse D II (m. Deckschotter) \\
\hline & & & Terrasse D I (Biburger T.) & Terrasse D I (o. Deckschotter) \\
\hline \multirow{5}{*}{$\begin{array}{l}\text { Biberkaltzeiten- } \\
\text { Komplex }\end{array}$} & Terrasse B V & Terrasse B V & Terrasse B V & \\
\hline & Terrasse B IV & Terrasse BIV & Terrasse BIV & \\
\hline & Terrasse B III & Terrasse B III & & Terrasse B III (Hochschotter) \\
\hline & Terrasse B II & Terrasse B II & Terrasse B II & \\
\hline & Terrasse B I (Hochfirst) & Terrasse B I (Morgenstelle) & & \\
\hline
\end{tabular}

Basis $15 \mathrm{~m}$ unterhalb der Terrasse D III, womit die Zusamplatte eine jüngere Akkumulation darstellt. Da bisher keine zwischenliegenden Terrassen zur D III gefunden wurden, kann die Zusamplatte als Terrasse D IV bezeichnet werden.

Die Abgrenzung zwischen biber- und donauzeitlichen Akkumulationen kann im Untersuchungsgebiet in Ermangelung chronostratigraphisch deutbarer Befunde nur aufgrund paläogeographischer Kriterien erfolgen. Die Grenze wurde nach der Ablagerung der Staudenplatten-Schotter gelegt, da sich anschließend der Wechsel der Iller von einem Tal über Mindelheim-Augsburg in ein weiter nordwestlich gelegenes vollzog. Für diesen Talwechsel war ein Durchbruch durch die Donau-Iller-Wasserscheide bei Memmingen nötig (Löscher \& Münzing \& TIllmanns 1978). Mit den vermutlich vom Lech abgelagerten Terrassen D I bis D III gleichzeitig entstandene Ablagerungen von der Iller ließen sich bislang nicht finden. Bis Alterseinstufungen der fraglichen Terrassen vorliegen, kann die Grenze Donau- zu Biberkaltzeiten-Komplex nur aufgrund vorstehender regionaler flußgeschichtlicher Kriterien gezogen werden.

\section{Flußgeschichtliche Entwicklung des Illergletscher-Vorlandes während des ältesten Pleistozäns}

Im ausgehenden Pliozän wurde das Landschaftsbild Bayerisch Schwabens von Molassekuppen geprägt, die von Alpenflüssen durchzogen wurden. Durch die nachfolgende glaziale und glazifluviatile Aktivität ist von der Gestalt dieser Landschaft nichts überliefert. Während der Biberkalt- zeit ist von einer alpinen Vergletscherung ohne Vorlandvereisung auszugehen, denn die sedimentäre Ausprägung der aus dieser Zeit erhaltenen Terrassen zeugt von starken Schwankungen der Wasserführung mit zeitweise großen Wassermassen, die auch grobes Sediment über mehrere zehner Kilometer zu transportieren vermochten. Zudem weisen die großen Mengen groben Verwitterungsschuttes, der die Terrassen aufbaut, auf verringerte Vegetationsbedeckung in den alpinen Tälern und erhöhte physikalische Verwitterungsintensität. Die aus den Zentralalpen stammenden Kristalline belegen Transfluenzen, wie sie bei einem Eisstromnetz innerhalb der Alpen zu erwarten sind.

Gemäß der Ausrichtung der Terrassengrenzen und der Gerölleinregelungen verlief das Illertal während der Biberkaltzeit von Kempten über Mindelheim und Augsburg zur Donau, die nördlich der Lechmündung durch die heutige Wellheimer Talung strömte (SCHAefer 1966 [Abb. 5]). Begrenzt wurde es von Tertiärrücken als Wasserscheiden zum Rhein im Westen und Lech im Südosten, dessen Verlauf durch fehlende Überlieferung jedoch hypothetisch bleiben muß. Die von SCHEuENPFlug (1991) angenommene Wasserscheide entlang des Südostrandes der Staudenplatte kann in dieser Form jedoch nicht bestanden haben, da sie die Grenzen der biberzeitlichen Terrassen schräg durchschneidet. Die Fließrichtung nach Nordosten stellt dabei möglicherweise einen Hinweis auf den tertiären Talverlauf dar, der von den biberzeitlichen Iller-Schmelzwässern benutzt wurde. Durch wahrscheinlich klimatische Schwankungen kam es zum fünfmaligen Wechsel 


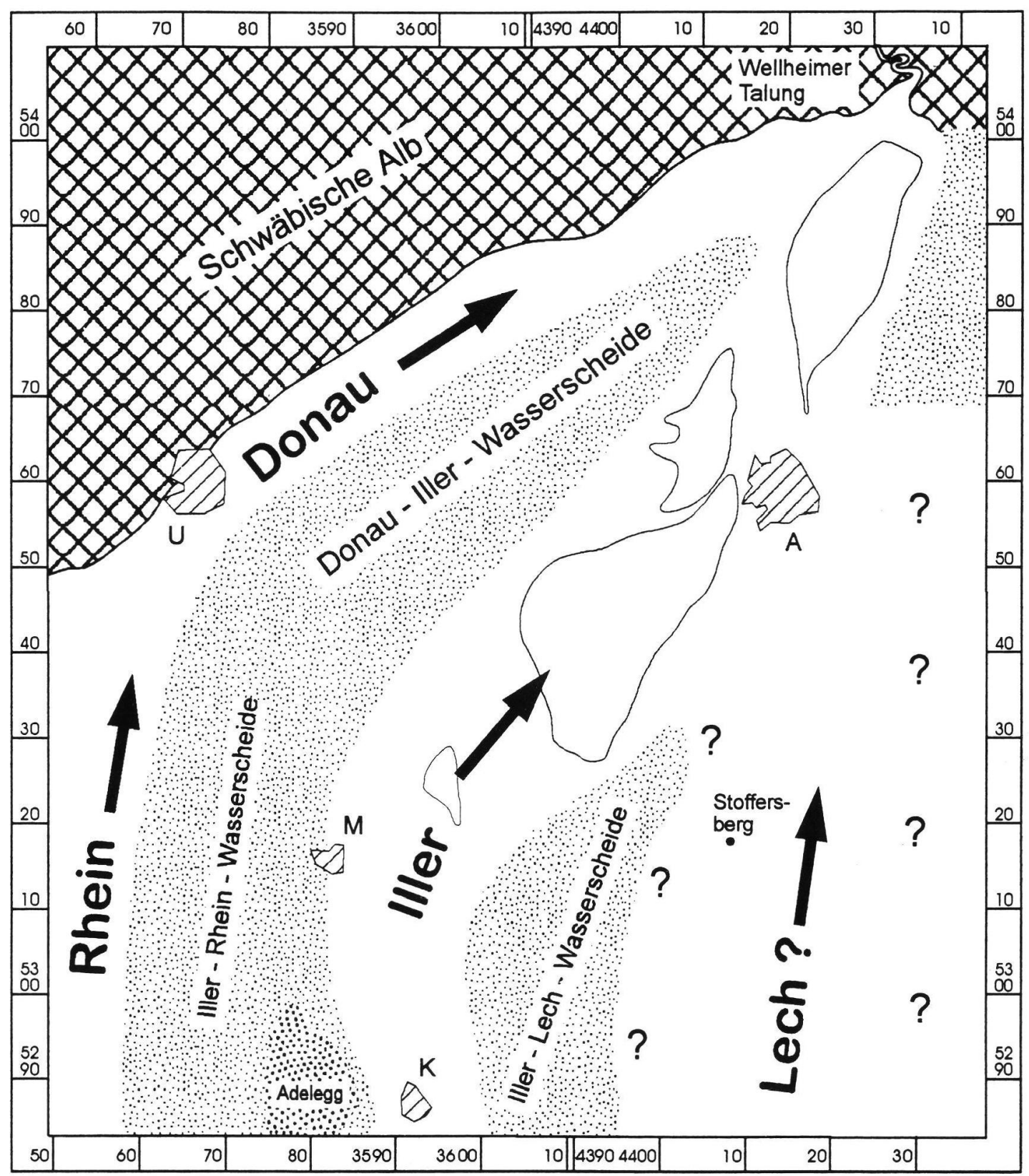

Abb. 5: Paläogeographie der Biberkaltzeit im Illergletscher-Vorland.

Fig. 5: Palaeogeographic scetch of Biberglaciation in Illerglacier foreland.

von Akkumulation und Erosion, die jeweils zu einer Einschneidung der Iller in ihre nächst ältere Ablagerung und zu nachfolgender Aufschotterung einer Terrasse führte. Die Terrassen B I bis B V stammen aus dieser Zeit und lassen auf ein anfangs bis $30 \mathrm{~km}$ breites Tal schließen.

Die Ausräumung eines solch breiten Tales kann durch die große Erosionskraft der schuttbeladenen Iller mit unsteter Wasserführung und star- kem Gefälle in Verbindung mit dem unkonsolidierten Untergrund erklärt werden. Unter diesen Bedingungen resultiert nach Rezentbeobachtungen ein lateral instabiler Fluß mit der Tendenz, ein großes Verhältnis von Talbreite zu durchschnittlicher Wassertiefe herauszubilden (BRIDGE 1993), was bei Vorhandensein leicht erodierbarer Ufer eine starke Ausweitung des Tales zur Folge hatte. 
Die Terrassenschotter bedeckten nach den vorliegenden Befunden auch das Dinkelscherbener Becken (Abb. 1), das heute inmitten der MindelLech-Platte eingetieft ist. Es wurde von GrauL (1949) als vormalige ältestpleistozäne Altwasserscheide gedeutet, welche durch Reliefumkehr ausgeräumt worden sei. Aus geometrischen Gründen ist es höchst unwahrscheinlich, daß das einstige Nordwest-Ufer der Iller im Süden um das Dinkelscherbener Becken herumgeführt hat, dann rechtwinklig nach Norden zum Staufenberg geschwenkt und anschließend erneut in rechtem Winkel nach Nordosten verlaufen sei. Die vorliegenden Ergebnisse sprechen dafür, daß das Becken durch die Zusam als einer der autochthonen Bäche aus der Terrasse B V ausgeräumt wurde. Diese Bäche entwässerten die biberzeitlichen Terrassen, nachdem die Iller sie verlassen hatte (Becker-Haumann im Druck). Die Bäche auf der heutigen Staudenplatte mündeten nach der Biberkaltzeit in einen Vorfluter, der auf der trockengefallenen Terrasse B V nach Nordosten floß und dessen letzten Rest die heutige mittlere Schmutter in ihrem Laufabschnitt zwischen Gessertshausen und Westheim darstellt (BeCKer-Haumann 1995 c). Nach Ablagerung der donauzeitlichen Zusamplatte wurde sie dem generellen Oberflächengefälle der Schotter entsprechend nach Norden entwässert, wobei sich Quellgebiete einiger Bäche (die heutigen Bäche Laugna, Roth und der Unterlauf der Zusam bis etwa Dinkelscherben) an der Steilkante zur 15 bis $20 \mathrm{~m}$ höher liegenden Terrasse B V der Staudenplatte befanden. Sie weiteten sich zu Quellkesseln aus, wodurch die untere Zusam den Vorfluter auf der Terrasse B V anzapfte. Die sprunghaft angestiegene Wasserführung und der einsetzende Gefällsausgleich bewirkten die flächenhafte Ausräumung des Beckens. Aufgrund von periglazial-fluviatilen Schottern der Schmutter läßst sich die Anzapfung der heutigen oberen durch die untere Zusam auf die Zeit zwischen Ablagerung der drittletzten (mindelzeitlich ?) und viertletzten (günzzeitlich ?) Schmutterterrasse festlegen.

Nach Entstehung der biberzeitlichen Terrassen kam es zur Ablagerung der drei im unteren Lechtal verbreiteten Terrassen D I bis D III aus südlicher Richtung. Die nächst jüngere fluviatile Akkumulation stellt dann der ZusamplattenSchotter D IV dar, der die am weitesten verbreitete Terrassenakkumulation auf der Iller-Lech-Platte bildet.

\section{Schriftenverzeichnis}

Aktas, A. \& Becker-Haumann, R. (1991): Zur Stratigraphie der südlichen Staudenplatte. - Geolog. Institut der Univ. zu Köln, Sonderveröff., 82: 9-18, 5 Abb. Köln.

Bachmann, G. \& Müller, M. (1996): Die Entwicklung des süddeutschen Molassebeckens seit dem Variszikum: Eine Einführung. - Z. geol. Wiss., 24: 3-20, 15 Abb.; Berlin.

Becker-Haumann (1995 a): Zur Korrelation der Aindlinger Terrassentreppe mit der Mindel-Lech-Platte. Geologica Bavarica, 99: 165-178, 8 Abb., 1 Tab., 2 Beil.; München.

- (1995 b): Zur Flußgeschichte der ältestpleistozänen Iller. - Geolog. Institut der Univ. zu Köln, Sonderveröff., 98: 168 S., 70 Abb., 10 Tab.; Köln.

- $(1995$ c): Die Periglazialschotter der mittleren Schmutter bei Augsburg. - Jber. Mitt. oberrhein. geol. Ver., N.F., 77: 329-349, 6 Abb., 2 Tab.; Stuttgart.

- (1996): Die Petrologie der kristallinen Iller-Gerölle (bayerisches Alpenvorland). - Aufschluss, 46: 13-28, 10 Abb.; Heidelberg.

- (1997): Der Arlesrieder Schotter - Ein weiterer Beleg für die biberzeitliche Iller in Bayerisch Schwaben. Geolog. Institut der Univ. zu Köln, Sonderveröff., 114: 59-75, 5 Abb., 2 Tab.; Köln.

- (im Druck): Die Entstehung des Dinkelscherbener Beckens - Ein Beitrag zur Landschaftsgeschichte Bayerisch-Schwabens. - Mitt. Geogr. Ges. München.

Bofnigk, W., Brelie, G. v. D., Brunnacker, K., Koci, A. Schlickum, W.R. \& STrauch, F. (1974): Zur PliozänPleistozän-Grenze im Bereich der Ville (Niederrheinische Bucht). - Newsl. Stratigr., 3: 219-241, 7 Fig. Leiden.

BRIDGE, J. (1993): The interaction between channel geometry, water flow, sediment transport and deposition in braided rivers. - In: Best, J. \& BRISTOW, C. (Hrsg.): Braided Rivers, Geological Society Special Publication, 75, 13-71, 26 Abb.; London.

Doppler, G. (1989): Zur Stratigraphie der nördlichen Vorlandsmolasse in Bayerisch Schwaben. - Geologica Bavarica, 94: 83-133, 25 Abb., 4 Tab.; München.

EBERHARD, M. (1987): Entwicklung von Sedimentation, Flora, Fauna, Klima und Relief von Mittelmiozän bis Quartär zwischen Arlberg (Vorarlberg/Tirol) und Adelegg (Allgäu). - Diss. der Eidgen. TH Zürich: 242 S., 50 Abb., 23 Abb., 6 Taf.; Zürich.

EBERL, B. (1930): Die Eiszeitenfolge im nördlichen Alpenvorland. - 427 S., 19 Abb., 2 Taf., 1 Kte.; Augsburg.

Ellwanger, D., Fejfar, O. \& Koenigswald, W. v. (1994): Die biostratigraphische Aussage der Arvicolidenfauna vom Uhlenberg bei Dinkelscherben und ihre morpho- und litostratigraphischen Konsequenzen. - Münch. Geowiss. Abh., A 26: 173-191, 24 Abb.; München

FERGUSON, R. (1993): Understanding braiding processes in gravel-bed rivers: progress and unsolved problems. - In: Best, J. \& Bristow, C. (Hrsg.): Braided Rivers, Geological Society Special Publication, 75, 73-87, 6 Abb.; London. 
Fezer, F. (1969): Tiefenverwitterung circumalpiner Pleistozänschotter. - Heidelb. Geogr. Arb., 24: 144 S., 90 Fig., 4 Abb., 1 Tab.; Heidelberg.

Filzer, P. \& Scheuenpflug, L. (1970): Ein frühpleistozänes Pollenprofil aus dem nördlichen Alpenvorland. - Eisz. u. Geg., 21: 22-32, 3 Abb.; Öhringen.

Graul, H. (1943): Zur Morphologie der Ingolstädter Ausräumungslandschaft. - Forsch. zur dt. Landeskde., 43: 114 S., 17 Abb., 8 Ktn.; Leipzig.

- (1949): Zur Gliederung des Altdiluviums zwischen Wertach-Lech und Flossach-Mindel. - Naturforsch. Ges. Augsburg, 2. Bericht: 3-31, 2 Abb., 3 Tab.; Augsburg.

HabBe, K. A. \& Rögner, K. (1989): The Pleistocene Iller Glaciers and their Outwash Fields. - Catena Supp., 15: 311-328, 4 Abb.; Cremlingen.

HANTKE, R. (1989): Kühl- und kaltzeitliche Klimaindizien im Jungtertiär der Schweiz, des Allgäus und einiger Nachbargebiete. - Geologica Bavarica, 94: $447-$ 457, 1 Abb.; München.

- (1993): Flußgeschichte Europas. - 459 S., 242 Abb.; Stuttgart (Enke).

Jerz, H., Stephan, W., Streit, R. \& Weinig, H. (1974): Geologische Übersichtskarte des Iller-Mindel-Gebietes 1:100.000. - Bayer. Geol. Landesamt: 37 S., 1 Beil., 1 Kte.; München

Krutzsch, W. (1988): Kritische Bemerkungen zur Palynologie und zur klimastratigraphischen Gliederung des Pliozäns bis tieferen Altpleistozäns in Süd-, Südwest-, Nordwest- und pro parte Mitteleuropa sowie die Lage der Pliozän/Pleistozän-Grenze in diesem Gebiet. - Quartärpal., 7: 7-51, 2 Tab.; Berlin.

Lemcke, K. (1988): Das bayerische Alpenvorland vor der Eiszeit. Geologie von Bayern I. - 175 S., 71 Abb., 1 Tab., 2 Taf.; Stuttgart.

Löscher, M. (1976): Die präwürmzeitlichen Schotterablagerungen in der nördlichen Iller-Lech-Platte. Heidelb. Geogr. Arb., 45: IX+157 S., 26 Abb., 4 Tab., 8 Taf., 4 Ktn., 17 Beil.; Heidelberg.

- Münzing, K. \& Tillmanns, W. (1978): Zur Paläogeographie der nördlichen Iller-Lech-Platte und zur Genese ihrer Schotter im Altpleistozän. - Eisz. u. Geg., 28: 68-82, 9 Abb.; Öhringen.

Penck, A. \& Brǘckner, E. (1909): Die Alpen im Eiszeitalter. - 3 Bde. 1199 S., 156 Abb., 30 Taf., 19 Ktn,; Leipzig.

RÄHLE, W. (1995): Altpleistozäne Molluskenfaunen aus den Zusamplattenschottern und ihrer Flußmergeldecke vom Uhlenberg und Lauterbrunn (Iller-LechPlatte, Bayerisch Schwaben). - Geologica Bavarica, 99: 103-117, München

Reichelt, G. (1961): Über Schotterformen und Rundungsgradanalyse als Feldmethode. - Petermanns geogr. Mitt., 105: 15-24, 7 Fig.; Gotha.

Rögner, K. (1979): Die glaziale und fluvioglaziale Dynamik im östlichen Lechgletschervorland - Ein Beitrag zur präwürmzeitlichen Pleistozänstratigraphie. - Heid. geogr. Arb., 49: 67-138, 12 Ktn., 12 Fig., 5 Prof., 5 Abb.; Heidelberg.

- (1986): Genese und Stratigraphie der ältesten Schotter der südlichen Iller-Lechplatte (BayerischSchwaben). - Eisz. u. Geg., 36: 111-119, 2 Abb., 3 Tab.; Hannover.

SchaEfEr, I. (1953): Die donaueiszeitlichen Ablagerungen an Lech und Wertach. - Geologica Bavarica, 19: 13-64, 15 Abb.; München.
- (1957): Erläuterungen zur Geologischen Karte von Augsburg und Umgebung 1:50.000. - Bayerisches Geologisches Landesamt: 92 S., 4 Abb., 1 Kte., 2 Beil.; München.

- (1966): Der Talknoten von Donau und Lech. - Mitt. geogr. Ges. München, 51: 59-111, 11 Abb.; München.

Scheuenpflug, L. (1986): Die altpleistozäne Hauptabflußrichtung der Gewässer in der Iller-Lech-Platte (Bayerisch Schwaben). - Jber. Mitt. oberrhein. geol. Ver., N.F., 68: 189-195, 1 Abb.; Stuttgart.

- (1991): Die frühpleistozäne Augsburger Altwasserscheide am Ostrand der Iller-Lech-Platte (süddeutsches Alpenvorland, Bayern). - Eisz. u. Geg., 41: 4755, 3 Abb.; Hannover.

- (1994): Die ältesten Schotter der Staufenbergserie in der nordöstlichen Iller-Lech-Platte Bayerisch Schwabens (Ältestpleistozän, älterer Teil der Biberkaltzeitengruppe) - Richtigstellungen und Ergänzungen. - Jber. Mitt. oberrhein. geol. Ver., N.F., 76: 261-271, 1 Abb., 2 Tab.; Stuttgart.

Schiemenz, S. (1960): Fazies und Paläogeographie der Subalpinen Molasse zwischen Bodensee und Isar. Beih. Geol. Jb., 38: 119 S., 6 Taf., 23 Abb.; Hannover.

SCHRÖDER, J. \& DeHM, R. (1951): Die Molluskenfauna aus der Lehm-Zwischenlage des Deckenschotters von Fischach, Kreis Augsburg. - Geologica Bavarica, 6: 118-120; München.

SinN, P. (1971): Zur Ausdehnung der Donau-Vergletscherung im schwäbischen Alpenvorland. - Eisz. u. Geg., 22: 188-191, 1 Abb.; Öhringen.

- (1972): Zur Stratigraphie und Paläogeographie des Präwürm im mittleren und südlichen Illergletschervorland. - Heidelb. Geogr. Arb., 37: 160 S., 13 Abb., 11 Tab., 21 Fig., 12 Prof., 5 Ktn.; Heidelberg.

Strattner, M. \& Rolf, C. (1995): Magnetostratigraphische Untersuchungen an pleistozänen Deckschicht-Profilen im bayerischen Alpenvorland. Geologica Bavarica, 99: 55-101; München.

Tillmanns, W. (1980): Zur plio-pleistozänen Flußgeschichte von Donau und Main in Nordostbayern. Jber. Mitt. oberrhein. geol. Ver., N.F., 62: 199-205, 3 Abb.; Stuttgart.

- Brunnacker, K. \& Löscher, M. (1983): Erläuterungen zur Geologischen Übersichtskarte der Aindlinger Terrassentreppe zwischen Lech und Donau 1:50.000. - Geologica Bavarica, 85: 31 S., 9 Abb., 1 Beil.; München.

- KočI, A. \& Brunnacker, K. (1986): Die Brunhes/Matuyama-Grenze in Roßhaupten (Bayerisch Schwaben). - Jber. Mitt. oberrhein. geol. Ver., N.F., 68: 241-247, 3 Abb.; Stuttgart.

Weidenbach, F. (1974): Über Nagelfluh, narbige und hohle Gerölle in pleistozänen Ablagerungen. Heid. geogr. Arb., 40: 331-340, 6 Abb.; Heidelberg.

WINTER, K.-P. (1968): Die Untere Mittelterrasse im Südteil der Niederrheinischen Bucht. - Geolog. Institut der Univ. zu Köln, Sonderveröff., 15: 138 S., 49 Abb., 5 Tab.; Köln.

ZAGWIJN, W. H. (1985): An outline of the Quaternary stratigraphy of the Netherlands.- Geol. en Mijnbo., 64: 17-24, 6 Fig.; Dordrecht.

Manuskript eingegangen am: 27. Juni 1997 